\title{
BMJ Open Is premature birth an environmental sensitivity factor? A scoping review protocol
}

Francesca Lionetti, ${ }^{1}$ Antonio Dellagiulia (1) , ${ }^{2}$ Giulia Prudentino, ${ }^{2}$ Maria Spinelli, ${ }^{1}$ Michael Pluess, ${ }^{3}$ Mirco Fasolo ${ }^{1}$

To cite: Lionetti F, Dellagiulia A, Prudentino $\mathrm{G}$, et al. Is premature birth an environmental sensitivity factor? A scoping review protocol. BMJ Open 2021;11:e047015. doi:10.1136/ bmjopen-2020-047015

- Prepublication history and additional supplemental material for this paper are available online. To view these files, please visit the journal online (http://dx.doi.org/10.1136/ bmjopen-2020-047015)

Received 18 November 2020 Accepted 13 October 2021

\section{Check for updates}

(c) Author(s) (or their employer(s)) 2021. Re-use permitted under CC BY-NC. No commercial re-use. See rights and permissions. Published by BMJ.

${ }^{1}$ Department of Neurosciences, Imaging and Clinical Sciences, Gabriele d'Annunzio University of Chieti and Pescara, Chieti,

Italy

${ }^{2}$ Psychology, Salesian Pontifical University, Roma, Italy

${ }^{3}$ Department of Biological and Experimental Psychology, Queen Mary University of London, London, UK

Correspondence to Dr Francesca Lionetti; francesca.lionetti@unich.it

\section{ABSTRACT}

Introduction Globally, around $10 \%$ of children are born preterm and are more at risk of negative developmental outcomes. However, empirical evidences and theoretical reasoning also suggest that premature birth can be a susceptibility factor, increasing sensitivity to the environment for better and for worse. Because available findings are controversial, with the current scoping review we will explore if, based on the available literature, preterm birth can be seen as an environmental sensitivity (ES) factor. In doing so, we will consider a series of moderating variables, including the level of prematurity, the type of environment and the outcome investigated. Methodological aspects, as the type of measures used and study design, will be considered.

Methods and analysis The scoping review will be conducted following the Joanna Briggs Institute Methodology guidelines. The report will follow the Preferred Reporting Items for Systematic Reviews and Meta-Analyses extension for Scoping Reviews checklist. We will perform the search between 15 January 2022 and 1 February 2022. Data will be chartered by independent reviewers.

Ethics and dissemination Ethical approval is not required, as primary data will not be collected. This scoping review will be the first to explore whether prematurity is associated with an increased ES. This review can have important implications for tailoring prevention and intervention programmes. Results will be published in a peer-reviewed journal.

\section{INTRODUCTION}

Preterm birth, which is the birth before the end of the 37 th gestational week, or less than 259 day from the beginning of the last menstrual period, ${ }^{1}$ is an important public health concern: it involves around $11 \%$ of births, with approximately 15 million of neonates estimated to be born preterm each year. ${ }^{2}$ Advancement in obstetric and neonatal care have decreased the risk of premature infant death, ${ }^{3}$ nevertheless, preterm delivery and its complications are the leading cause of neonatal morbidity and mortality. ${ }^{4}$ Although most preterm babies survive, preterm birth is associated with an increased risk of serious

\section{Strengths and limitations of this study}

This is the first scoping review on premature birth and environmental sensitivity.

- We will follow Joanna Briggs Institute and Preferred Reporting Items for Systematic Reviews and MetaAnalyses extension for Scoping Reviews guidelines.

- We will include articles written in four different languages.

- We will not evaluate the quality of evidence, as this would be beyond the aim of scoping reviews.

- We might not be able to provide a unique answer to our research question, but mixed findings will be discussed.

medical conditions, such as cerebral palsy, mental retardation, blindness or low vision, hearing loss and epilepsy, and of higher rates of behavioural disorders and socioemotional problems. ${ }^{5}$ Several studies reported preterm children to have a higher rate of behavioural issues, ${ }^{67}$ poorer performance on cognitive tasks $^{8-10}$ and a greater likelihood of fine and gross motor skill impairment. ${ }^{9} 1112$ For its negative consequences on children's concurrent adjustment and subsequent development, preterm birth has been typically viewed as a vulnerability factor. However, more recent theoretical reasoning and empirical evidences also suggest that preterm birth can be more broadly viewed as an environmental sensitivity factor, ${ }^{13}{ }^{14}$ increasing individual susceptibility to environmental stimuli, both negative and positive. ${ }^{15}$ For example, empirical evidences suggest that premature children are more susceptible to the quality of early parental emotional adjustment, presenting more positive social outcomes at age 12 months compared with full-term births when experiencing a positive rearing environment. ${ }^{16}$ Similar findings have been reported in relation to cognitive outcomes, with very preterm infants, born before 30 weeks of gestational age, benefiting more than 
moderate preterm and late preterm children from the experience of early positive parenting for what pertains cognitive scores at age 3 years. ${ }^{17}$ Taken together, these findings suggest that preterm infants are more developmentally responsive to their environment. Considering that prenatal stress is a risk factor for preterm birth and low birth weight, ${ }^{18}$ and in line with empirical evidences and theoretical reasoning suggesting that there might be a prenatal programming of postnatal plasticity driven by higher levels of stress experienced during pregnancy, ${ }^{19-24}$ we might indeed consider preterm birth as a factor related to an increased susceptibility to the environment, for better and for worse. However, it has to be acknowledged that other empirical evidences ${ }^{25}$ did not provide support to prematurity as a susceptibility factor, and other data reported prematurity to be a vulnerability factor only. ${ }^{16}$ It might be that preterm birth is a susceptibility factor for some developmental outcomes but not for others (eg, for behavioural problems but not in relation to cognitive development), or that other variables, including the prematurity level and the type of environment considered (eg, parental care or quality of child-care services), are able to explain mixed findings reported in the literature. Investigating if preterm birth represents a susceptibility factor, and in which condition this is more likely to be the case, could allow to better tailor intervention and prevention programmes. ${ }^{26}$ Also, from a theoretical perspective, it would provide more information on what variables should be considered when exploring factors contributing to the development of children born preterm. Given the high rate of preterm births, and the lack of consensus in the scientific literature on the concept of prematurity as a susceptibility factor to environmental influences, it is critical to examine this area further.

\section{Scoping review objectives}

In order to explore whether premature birth can be seen as a factor increasing individual levels of sensitivity to the environment, we will systematically search for the scientific literature investigating the interplay between preterm birth and the quality of the environment in predicting concurrent adjustment and longitudinal development in preterm children. In other words, we aim to explore whether premature infants are more likely to be more susceptible to the impact of their rearing environment, for better and for worse, and if this appears to be true irrespective of the type of the environment and of the outcome considered, or if some specificities and patterns there exist. In doing so, we will assume as a theoretical framework of our scoping review the environmental sensitivity theory, ${ }^{13}$ which postulates that individual differ in response to environmental stimuli and integrates different theoretical contributions and model on the individual environment interplay, including differential susceptibility, ${ }^{1527}$ biological sensitivity to the context, ${ }^{28}$ sensory processing sensitivity, ${ }^{29}$ diathesis stress $^{30}$ and vantage sensitivity. ${ }^{14}{ }^{26}$ According to the environmental sensitivity meta-framework, a significant minority of the population, around $25 \%-30 \%$, is highly sensitive to stimuli. ${ }^{3031}$ Markers of this increased sensitivity have been considered genetic variants, ${ }^{32}$ phenotypical traits ${ }^{29} 303334$ and physiological variables, ${ }^{35}$ which are influenced from both genetic and environmental factors contributing to the calibration of biological stress response systems.

In reviewing existing articles, we will provide information on children's outcomes and environmental variables to explore whether the susceptibility effect applies to all developmental psychology domains or only to some of these, and whether individual susceptibility of preterm infants is manifested in response to all environmental contexts or only in relation to some of these. In addition, we will consider the individual variable of gestational age at birth, and methodological aspects including the research design, timing of assessment of the outcome variable(s) (ie, early infancy, toddlerhood, preschool years, middle childhood, adolescence), and measures of the environment and outcomes (observational, questionnaires, qualitative measure, standardised test).

To the best of our knowledge, according a preliminary research conducted the 26 of October 2020 on Scopus, PubMed and Web of Science, previous scoping review are not available on this topic.

\section{METHODS AND ANALYSIS}

In order to map the available studies on prematurity and environmental sensitivity, we will conduct a scoping review following the Joanna Briggs Institute Methodology (JBI) guidelines for scoping reviews. ${ }^{36}$ The scoping review report will follow the Preferred Reporting Items for Systematic Reviews and Meta-Analyses (PRISMA) extension for Scoping Reviews checklist. ${ }^{37}$

\section{Studies eligibility criteria}

Studies investigating preterm children socio-emotional and cognitive development (eg, emotion regulation, executive functioning, peer relationships) will be considered. According to the objectives of the review, a measure of environmental quality (eg, parenting, child-care services quality, intervention) should be reported in, together with an estimation of its impact on children's adjustment.

We will include papers focused on very preterm, moderate preterm and late preterm children, as well as low birth weight children, considering both spontaneous and inducted birth. Children up to early adolescents (till 14 years old) will be included.

\section{Participants/patient and public involvement}

Patients are not involved in this protocol.

\section{Concept}

The overarching interest of this scoping review is to explore whether premature birth represents a factor associated with an increased environmental sensitivity, ${ }^{13}$ to stimuli, and to identify candidate moderators eventually responsible for differences among studies. 


\section{Context}

We have no reason to limit the context to a specific geographical, social, cultural context; studies from any contextual setting will be considered.

\section{Study types}

The review will include primary published research studies with empirical findings (including, but not limited to, cross-sectional, longitudinal, experimental and single case studies) based on quantitative, qualitative as well as mixed-methods methodology. We will consider also theoretical papers for critically discussing findings and more generally the breadth of research conducted thus far on the topic.

\section{Search strategy}

According JBI guidelines, we will follow a three steps strategy. First, we will search in two out of the three identified datasets, namely PubMed and Scopus, using the keywords presented in table 1.

We will screen title and abstract in order to refine, if necessary, our keywords. In the second step, we will perform the search in all the three databases, including Web of Science, with the identified keywords. Third, starting from the reference list of the sources selected from full text and included in the review we search for additional sources.

All the records will be imported in Mendeley and all duplicates will be deleted before the screening stage. Search will be conducted on all available sources till the date of search implementation. We will consider sources in Italian, English, French and Spanish due to authors language knowledge. Studies in other languages will be included if a version in the language considered could be provided. We aim to search across databases, in accordance with the plan detailed above, between 15 January 2022 and 1 February 2022.

In order to verify the feasibility of the current scoping review and of the proposed keywords, we conducted a pilot search in 17 May 2021, and a reasonable number of studies, that is, not too small to limit the opportunity of critically discussing the topic of interest, and not too broad to avoid any type of synthesis, was identified. More specifically, we identified 119 papers in PubMed (searching in the Title/abstract), 399 in Web of Science (searching in all database for the title/abstract/keywords/keywords plus field) and 304 in Scopus (searching in the title/ abstract/keywords field). No other exclusion criteria were set expect for language where applicable (English, French, Italian and Spanish in PubMed and Scopus). Full strings of this pilot search are provided in online supplemental material.

\section{Study selection}

In the screening phase, two reviewers, using the eligibility criteria, will independently screen the sources identified based on title and abstract. In the second stage, other two independent reviewers will review full-text
Table 1 Keywords for the first step of the search strategy

\begin{tabular}{|c|c|c|}
\hline \multicolumn{3}{|l|}{ Keywords } \\
\hline Prematurity & $\begin{array}{l}\text { Enviromental } \\
\text { sensitivity }\end{array}$ & Outcome \\
\hline $\begin{array}{l}\text { Preterm } \\
\text { OR } \\
\text { Pre-term } \\
\text { OR } \\
\text { Prematur* } \\
\text { OR } \\
\text { Low birthweight }\end{array}$ & $\begin{array}{l}\text { Differential } \\
\text { Susceptibility } \\
\text { OR } \\
\text { Biological } \\
\text { Sensitivity to the } \\
\text { Context } \\
\text { OR } \\
\text { Environmental } \\
\text { Sensitivity } \\
\text { OR } \\
\text { Diathesis Stress } \\
\text { OR } \\
\text { Vantage Sensitivity } \\
\text { OR } \\
\text { Susceptib* } \\
\text { OR } \\
\text { Vulnerab* } \\
\text { OR } \\
\text { Plasticity }\end{array}$ & $\begin{array}{l}\text { Socio-emotional } \\
\text { development } \\
\text { OR } \\
\text { Socio-emotional } \\
\text { competence* } \\
\text { OR } \\
\text { Emotional } \\
\text { competence* } \\
\text { OR } \\
\text { Emotional } \\
\text { development OR } \\
\text { Social competence* } \\
\text { OR } \\
\text { Social development } \\
\text { OR } \\
\text { Peer relationship* } \\
\text { OR } \\
\text { Self-esteem } \\
\text { OR } \\
\text { Self regulation } \\
\text { OR Emotion } \\
\text { regulation } \\
\text { OR } \\
\text { Behav* problem* } \\
\text { OR } \\
\text { Language } \\
\text { development } \\
\text { OR } \\
\text { Executive functioning } \\
\text { OR } \\
\text { Cognitive* } \\
\text { development } \\
\text { OR } \\
\text { Cognitive abilit* } \\
\text { OR } \\
\text { Cognitive } \\
\text { competence* } \\
\text { OR } \\
\text { IQ } \\
\text { OR } \\
\text { Academic } \\
\text { achievement } \\
\text { OR }\end{array}$ \\
\hline
\end{tabular}

article. Disagreements in both stages will be solved by consensus between reviewer and eventually the involvement of a third reviewer. Prior to start the screening phase, a random selection of 25 sources (title/abstract) will be screened from a fourth reviewer and, if necessary, eligibility criteria refined until an agreement of $75 \%$ is achieved. Using a PRISMA flow diagram, ${ }^{38}$ we will report for each stage the number of studies excluded and the reason of the exclusion.

\section{Data extraction}

A draft of a data-charting table is reported in online supplemental material. A pilot study of charting table 
will be done on three studies by all the authors of the protocol. Adaptation of charting table following the pilot study or during the data extraction will be reported on the paper. Two independents reviewers will extract and check the data. More specifically, for each source, data will be extracted by one reviewer and checked by the other one. Disagreement will be solved by discussion and if necessary by a third reviewer.

\section{Data synthesis}

Results will be presented in a tabular form and a narrative format, following the aim of the review. We will report results considering specifically the type of environmental variable considered (eg, parenting quality vs other environments), the way the environmental and outcome variables have been assessed (eg, using questionnaires, observational measures), the type of outcome considered), the study design, the level of prematurity (ie, very preterm birth, moderate preterm birth, low birth weight) and the presence as well the type of control group (if any) involved. We will summarise findings considering if support is provided for non-enhanced susceptibility, for environmental sensitivity for better and for worse (in line with differential susceptibility model), for better (as in the vantage sensitivity framework) or for worse only (according to the diathesis-stress framework).

\section{ETHICS AND DISSEMINATION}

Preterm birth rate is currently estimated to be around $11 \%$. Preterm birth has been repeatedly reported as a risk factor for both physical, behavioural and psychological adjustment. However, empirical evidences over the last 10 years also suggest that premature birth can be seen as a plasticity factor, increasing not only children's vulnerability, but also positive responses to positive stimuli. However, there are contradictory findings too, and results do not only converge on the same conclusion. With the current review we aim to shed light on the interplay between the environment and the premature birth condition to explore if prematurity can be seen as an environmental sensitivity factor. Through the analysis of a series of moderators, including the type of the environment, the outcome and the study design, we aim to clarify in which condition this is more likely to occur. Identifying if preterm birth does indeed increase susceptibility to the environment, what types of environmental influences are more likely to strongly impact on preterm children's development, and what developmental outcomes are more likely to be more strongly influenced by the environment, could have important implications for better tailoring intervention and prevention programmes that target the preterm child and her environment. The results will be published through a peer-reviewed journal.

Contributors FL developed the main conceptual idea and with AD, GP and MS wrote the paper and defined the study protocol. MP and MF revised the final version of the manuscript.
Funding This work was supported by Programma Operativo Nazionale (PON)-AIM grant number PON-AIM 1811283-3 to G. d'Annunzio University.

Competing interests None declared.

Patient consent for publication Not applicable.

Provenance and peer review Not commissioned; externally peer reviewed.

Supplemental material This content has been supplied by the author(s). It has not been vetted by BMJ Publishing Group Limited (BMJ) and may not have been peer-reviewed. Any opinions or recommendations discussed are solely those of the author(s) and are not endorsed by BMJ. BMJ disclaims all liability and responsibility arising from any reliance placed on the content. Where the content includes any translated material, BMJ does not warrant the accuracy and reliability of the translations (including but not limited to local regulations, clinical guidelines, terminology, drug names and drug dosages), and is not responsible for any error and/or omissions arising from translation and adaptation or otherwise.

Open access This is an open access article distributed in accordance with the Creative Commons Attribution Non Commercial (CC BY-NC 4.0) license, which permits others to distribute, remix, adapt, build upon this work non-commercially, and license their derivative works on different terms, provided the original work is properly cited, appropriate credit is given, any changes made indicated, and the use is non-commercial. See: http://creativecommons.org/licenses/by-nc/4.0/.

\section{ORCID iD}

Antonio Dellagiulia http://orcid.org/0000-0003-1925-5743

\section{REFERENCES}

1 WHO. Who: recommended definitions, terminology and format for statistical tables related to the perinatal period and use of a new certificate for cause of perinatal deaths. modifications recommended by FIGO as amended October 14, 1976. Acta Obstet Gynecol Scand 1977;56:247-53.

2 Chawanpaiboon S, Vogel JP, Moller A-B, et al. Global, regional, and national estimates of levels of preterm birth in 2014: a systematic review and modelling analysis. Lancet Glob Health 2019;7:e37-46.

3 Crump C, Winkleby MA, Sundquist J, et al. Prevalence of survival without major comorbidities among adults born prematurely. JAMA 2019;322:1580-8.

4 Lawn JE, Cousens S, Bhutta ZA, et al. Why are 4 million newborn babies dying each year? Lancet 2004;364:399-401.

5 Moster D, Lie RT, Markestad T. Long-Term medical and social consequences of preterm birth. N Engl J Med 2008;359:262-73.

6 Arpino C, Compagnone E, Montanaro ML, et al. Preterm birth and neurodevelopmental outcome: a review. Childs Nerv Syst 2010;26:1139-49.

7 Delobel-Ayoub M, Arnaud C, White-Koning M, et al. Behavioral problems and cognitive performance at 5 years of age after very preterm birth: the EPIPAGE study. Pediatrics 2009;123:1485-92.

8 Anderson P, Doyle LW, Victorian Infant Collaborative Study Group. Neurobehavioral outcomes of school-age children born extremely low birth weight or very preterm in the 1990s. JAMA 2003;289:3264-72.

9 Baron IS, Weiss BA, Baker R, et al. Subtle adverse effects of late preterm birth: a cautionary note. Neuropsychology 2014;28:11-18.

10 Ionio C, Riboni E, Confalonieri E, et al. Paths of cognitive and language development in healthy preterm infants. Infant Behav Dev 2016;44:199-207.

11 Foulder-Hughes LA, Cooke RWI. Motor, cognitive, and behavioural disorders in children born very preterm. Dev Med Child Neurol 2003;45:97-103.

12 Sullivan MC, Hawes K. A decade comparison of preterm motor performance at age 4. Res Nurs Health 2007;30:641-54.

13 Pluess M. Individual differences in environmental sensitivity. Child Dev Perspect 2015;9:138-43.

14 Greven CU, Homberg JR. Sensory processing sensitivity-For better or for worse? Theory, evidence, and societal implications. In: The highly sensitive brain. Elsevier, 2020: 51-74.

15 Belsky J, Pluess M. Beyond diathesis stress: differential susceptibility to environmental influences. Psychol Bull 2009;135:885-908.

16 Gueron-Sela N, Atzaba-Poria N, Meiri G, et al. The caregiving environment and developmental outcomes of preterm infants: diathesis stress or differential susceptibility effects? Child Dev 2015;86:1014-30.

17 Shah PE, Robbins N, Coelho RB, et al. The paradox of prematurity: the behavioral vulnerability of late preterm infants and the cognitive 
susceptibility of very preterm infants at 36 months post-term. Infant Behav Dev 2013;36:50-62.

18 Shapiro GD, Fraser WD, Frasch MG, et al. Psychosocial stress in pregnancy and preterm birth: associations and mechanisms. $J$ Perinat Med 2013;41:631-45.

19 Davis EP, Snidman N, Wadhwa PD, et al. Prenatal maternal anxiety and depression predict negative behavioral reactivity in infancy. Infancy 2004;6:319-31.

20 Baibazarova E, van de Beek C, Cohen-Kettenis PT, et al. Influence of prenatal maternal stress, maternal plasma cortisol and cortisol in the amniotic fluid on birth outcomes and child temperament at 3 months. Psychoneuroendocrinology 2013;38:907-15.

21 Sharp H, Hill J, Hellier J, et al. Maternal antenatal anxiety, postnata stroking and emotional problems in children: outcomes predicted from pre- and postnatal programming hypotheses. Psychol Med 2015;45:269-83.

22 Pluess M, Belsky J. Prenatal programming of postnatal plasticity? Dev Psychopathol 2011;23:29-38.

23 Hartman S, Belsky J. Prenatal programming of postnatal plasticity revisited-And extended. Dev Psychopathol 2018;30:825-42.

24 Hartman S, Belsky J. Prenatal stress and enhanced developmental plasticity. J Neural Transm 2018;125:1759-79.

25 Hadfield K, O'Brien F, Gerow A. Is level of prematurity a risk/plasticity factor at three years of age? Infant Behav Dev 2017;47:27-39.

26 de Villiers B, Lionetti F, Pluess M. Vantage sensitivity: a framework for individual differences in response to psychological intervention. Soc Psychiatry Psychiatr Epidemiol 2018;53:545-54.

27 Belsky J. Variation in susceptibility to environmental influence: an evolutionary argument. Psychol Inq 1997;8:182-6.

28 Ellis BJ, Boyce WT, Belsky J, et al. Differential susceptibility to the environment: an evolutionary--neurodevelopmental theory. Dev Psychopathol 2011;23:7-28.
29 Lionetti F, Aron EN, Aron A, et al. Observer-rated environmental sensitivity moderates children's response to parenting quality in early childhood. Dev Psychol 2019;55:2389-402.

30 Lionetti F, Aron A, Aron EN, et al. Dandelions, TULIPs and orchids: evidence for the existence of low-sensitive, medium-sensitive and high-sensitive individuals. Trans/ Psychiatry 2018;8:24.

31 Pluess M, Assary E, Lionetti F, et al. Environmental sensitivity in children: development of the highly sensitive child scale and identification of sensitivity groups. Dev Psychol 2018;54:51-70.

32 Keers R, Coleman JRI, Lester KJ, et al. A genome-wide test of the differential susceptibility hypothesis reveals a genetic predictor of differential response to psychological treatments for child anxiety disorders. Psychother Psychosom 2016;85:146-58.

33 Slagt M, Dubas JS, van Aken MAG, et al. Sensory processing sensitivity as a marker of differential susceptibility to parenting. Dev Psychol 2018;54:543-58.

34 Greven CU, Lionetti F, Booth C, et al. Sensory processing sensitivity in the context of environmental sensitivity: a critical review and development of research agenda. Neurosci Biobehav Rev 2019;98:287-305.

35 Boyce WT. Differential susceptibility of the developing brain to contextual adversity and stress. Neuropsychopharmacology 2016;41:142-62.

36 Aromataris E, Munn Z. JBI Reviewer's Manual. JBI Rev Man 2019.

37 Tricco AC, Lillie E, Zarin W, et al. PRISMA extension for scoping reviews (PRISMA-ScR): checklist and explanation. Ann Intern Med 2018;169:467-73.

38 Moher D, Shamseer L, Clarke M. Preferred reporting items for systematic review and meta-analysis protocols (PRISMA-P) 2015 statement. Rev Esp Nutr Humana y Diet 2016;20:148-60. 